\title{
Physician Response to Medtronic's Position on the Use of Off-label Medications in the Synchromed Pump
}

n November of 2012, Medtronic sent an Urgent Medical Device Safety Notification to physicians who implant Medtronic Synchromed intrathecal infusion pumps warning them not to use unapproved (off-label) medications

in the SynchroMed pump. In this communication, Medtronic stated, "The use of unapproved drugs can lead to intermittent or permanent pump motor stalls which may be reported as a loss of or change in therapy. Therapy changes could potentially result in serious injury and/or death." This strongly-worded Medtronic Safety Notification has caused great consternation among implanting physicians because off-label (and hence unapproved) medications are commonly used in SynchroMed pumps and the majority of chronic pain patients require the use of these offlabel medications to achieve adequate pain relief or to control unacceptable drug induced side effects.

As national and international societies representing thousands of physicians who manage chronic pain patients with intrathecal drug delivery (IDD) using the Medtronic SynchroMed pump and catheter system, we are compelled to respond to Medtronic's stated position regarding the use of off-label medications in the SynchroMed pump. Today the vast majority of patients receiving continuous long term intrathecal therapy for pain are receiving compounded admixtures of medications defining this as the current standard of care. The need for this clinical approach of using compounded medication admixtures for IDD relates to the lack of commercially available preparations to replace the multitude of medication combinations commonly used by expert implanters. It is important to note that pump failure can occur for a variety of reasons and therefore vigilance on both the physician and patient's part are important whether on or off label medications are utilized.

\section{The issue:}

Patients who have implanted intrathecal pumps have by definition, extreme, intractable chronic pain that cannot be effectively treated with more conservative measures. Prior to pump implant, these patients are often taking high doses of oral opioids, which cause significant, untoward side effects and risk of death from overdose.

There are 4 Food and Drug Administration (FDA) approved medications for use in the Synchromed pump to treat pain and/or spasticity:

1. Infumorph: a manufactured form of preservative-free morphine

2. Prialt: a synthetic conopeptide N-type calcium channel blocker.

3. Lioresal and Gablofen, manufactured forms of baclofen, are also approved for use in the Synchromed pump to treat spasticity but not for pain management.
Each of these drugs is FDA approved only as monotherapy and there are no FDA approved drug admixtures. When either Infumorph or Prialt is used as a monotherapy, pain relief is often inadequate or intolerable side effects may occur. In view of the cost associated with performing randomized, controlled trials on intrathecal medications (and the hundreds of potential medication combinations), it is uncertain whether any other medications will ever be approved for use in the Synchromed pump. Nonetheless, off-label medications have been used safely and effectively in IDD for many years and continue to be used on a regular basis by many implanting physicians.

Many experienced implanters believe that intrathecal hydromorphone, fentanyl and other opioids are more effective, better tolerated, and may be safer than intrathecal morphine monotherapy in certain patients. Furthermore, many implanters believe that intrathecal drug admixtures which combine an opioid with a local anesthetic provide better pain relief at lower 
doses of each medication. Intrathecal opioids relieve pain by binding to intraspinal opioid receptors whereas local anesthetics block nerve conduction and there is substantial evidence in the medical literature that the effects of these two medications are synergistic when used for intrathecal pain relief. There is also scientific evidence to support the use of clonidine and other medications in intrathecal admixtures. After reviewing the existing scientific evidence, many implanting physicians use their best medical judgment and experience to choose compounded admixtures of off-label drugs in the SynchroMed pump for optimal pain control in their patients. This practice is described and supported with recommendations on dosing parameters in four landmark articles titled Polyanalgesia Consensus Statements (Bennet, et. al. 2000, Hassenbush et. Al 2003, Deer et. al. 2007, Deer et. Al 2012).

\section{The facts:}

Abrupt cessation of therapy is a known risk with intrathecal drug delivery for both approved and unapproved drugs and can happen for a variety of reasons including catheter dislodgment, catheter occlusion and pump failure. The fact that therapy cessation is an inherent risk in IDD requires vigilance on the part of caregivers and patients if this therapy is chosen. It is important to note that catheter mechanical failure is estimated to be greater than $10 \%$ over the life of a pump in some studies and would appear to be a more common cause for therapy cessation than pump failure.

Pump catheters may be more likely to occlude with unapproved drugs although there is insufficient data to quantify this risk. The ISPR registry data has substantial limitations and cannot determine length of pump exposure to off label medication, which drugs are most implicated in pump failure, what compounding characteristics contribute to an increased risk of pump failure (specific drug, concentration, $\mathrm{pH}$ etc.) and what clinical practices mitigate failure rates (flow rates, PTM, refill interval).

With regard to pump failure, approved and unapproved medications may cause pump corrosion and failure. According to the most recent analysis of data from the Medtronic ISPR database, the incidence of pump failure at 78 months post implant is $2.4 \%$ with approved drugs and $7.0 \%$ with unapproved drugs. The nature of the unapproved drugs cannot be clarified from the ISPR database other than to say the drugs were not Infumorph or Prialt used as monotherapy.
Therefore, the increased risk of pump failure with off-label drugs may be due in part to the use of highly concentrated admixtures of multiple drugs that are not compounded to optimal standards of purity and $\mathrm{pH}$.

The ISPR data shows that the overall risk of pump motor stall is low regardless of whether approved or unapproved drugs are used in the pump. After 5 years, pumps with approved drugs have a $2.4 \%$ failure rate compared to a $4.5 \%$ failure rate in pumps with unapproved drugs. After 5 years, $97.6 \%$ of pumps with approved drugs and $95.5 \%$ of pumps with unapproved drugs are functional. At end of battery life (@ 81 months), $97.6 \%$ of approved drug pumps are functional whereas $93 \%$ of unapproved drug pumps are functional.

There is a significant difference in the risks of pump and/or catheter failure in chronic pain patients treated with intrathecal opioids compared to spasticity patients treated with intrathecal baclofen. Therapy cessation with high dose baclofen for spasticity results in baclofen withdrawal, which is serious and occasionally fatal. Therapy cessation in pain patients receiving intrathecal opioids may result in unpleasant opioid withdrawal symptoms and return of baseline pain but is typically not life-threatening. Hospitalization is rarely required for therapy cessation resulting from intrathecal opioid withdrawal since withdrawal symptoms can be readily reversed with oral opioids and these medications can be continued for pain relief until elective pump/catheter revision surgery is undertaken.

Given the clinical need to use patient specific admixtures of intrathecal compounded medications for the treatment of chronic pain, there is a need for additional investigation as to the cause for the observed findings which led to this Medtronic safety notification, including further studies to develop guidelines on proper medication compounding and safe drug concentrations. We recommend to both pump manufacturers and FDA to widen the spectrum of approved medications for intrathecal delivery in these devices to reflect standard clinical practice and to remove additional liability from physician-prescribers.

\section{Recommended Actions for Implanting Physicians:}

Compounded intrathecal medications are in common use and represent the de facto standard of care. Peer reviewed literature is available regarding the accepted standards of safe use of intrathecal medica- 
tions both on and off label. It is reasonable, acceptable and typically necessary for physicians to use so called "off label" medications to effectively treat intractable chronic pain. To deprive patients of these medications will result in suboptimal pain relief and a decrease in quality of life and is not consistent with best practices or standard of care. In medical practice, it is reasonable, acceptable and sometimes necessary for physicians to use off label medications to effectively treat intractable disease and this is especially true for intrathecal drug delivery in patients with intractable chronic benign and cancer pain.

We make the following recommendations for the safe and effective use of off label medications in the Synchromed pump:

1. Disclose all risks of intrathecal drug delivery to the patient prior to trial and implant. Specifically address the risks, benefits and uncertainties associated with the use of off label intrathecal medications. Bring the patient into the decision making process when contemplating the use of off label medications in the pump.

2. Educate the patient about the signs and symptoms of withdrawal.

3. Review the Polyanalgesia Consensus Statement articles and other published scientific literature to determine which medications and medication admixtures are safe and effective for use in intrathecal drug delivery.

4. Be aware of published recommendations as to optimal dosing parameters, concentrations and admixture combinations.

5. Increased caution and vigilance should be exercised when using high dose baclofen as part of an off-label admixture for the treatment of pain. High dose baclofen is most often utilized as monotherapy in spasticity patients and should be used cautiously in pain patients in order to minimize the risk of serious baclofen withdrawal reactions.

6. Order off-label intrathecal medications and medication admixtures only from experienced, compounding pharmacies that compound intrathecal medications to recommended standards of $\mathrm{pH}$ and purity since both suboptimal acidity and impurities in the medication may be responsible for device malfunction. These pharmacies should follow USP 797 standards (or be similarly compliant for nonU.S. pharmacies).

7. When using boluses, caution should be exercised, especially in cases when using medications with potential vasomotor impact (i.e. - clonidine)

8. At each pump refill, history logs should be reviewed to look for unexpected motor stalls which may be a precursor to pump failure.

In summary, physicians adhering to these recommended actions are striking the optimal benefit to risk ratio for their patients. Awareness and vigilance is essential to minimize complications with intrathecal therapy whether on label or off-label medications are utilized.

Ali Rezai, MD

President

North American Neuromodulation Society

David Kloth, MD

President-Elect

North American Neuromodulation Society

Past-President and Board of Directors

American Society of Interventional Pain Physicians

Hans Hansen, MD

President

American Society of Interventional Pain Physicians

David Schultz MD

Past President and Board of Directors

American Society of Interventional Pain Physicians

Simon Thompson, MBBS

President

International Neuromodulation Society

Timothy Deer, MD

President-elect

International Neuromodulation Society 
